\title{
Color prediction modeling for five-channel CMYLcLm printing
}

\author{
Yuanyuan Qu, Paula Zitinski Elias and Sasan Gooran
}

\section{Linköping University Post Print}

\section{Tweet}

N.B.: When citing this work, cite the original article.

Original Publication:

Yuanyuan Qu, Paula Zitinski Elias and Sasan Gooran, Color prediction modeling for fivechannel CMYLcLm printing, 2014, Color Imaging XIX: Displaying, Processing, Hardcopy, and Applications, 901508-1-901508-11.

http://dx.doi.org/10.1117/12.2035736

Copyright: (C) 2014 SPIE-IS\&T

http://proceedings.spiedigitallibrary.org/

Postprint available at: Linköping University Electronic Press

http://urn.kb.se/resolve?urn=urn:nbn:se:liu:diva-106105 


\title{
Color prediction modeling for five-channel CMYLcLm printing
}

\author{
Yuanyuan $\mathrm{Qu}^{\mathrm{a}}$, Paula Zitinski Elias ${ }^{* \mathrm{a}}$ and Sasan Gooran ${ }^{\mathrm{a}}$ \\ ${ }^{a}$ Department of Science and Technology, Linköping University, Sweden
}

\begin{abstract}
In printing, halftoning algorithms are applied in order to reproduce a continuous-tone image by a binary printing system. The image is transformed into a bitmap composed of dots varying in size and/or frequency. Nevertheless, this causes that the sparse dots found in light shades of cyan (C) and magenta (M) appear undesirably noticeable against white substrate. The solution is to apply light cyan $(\mathrm{Lc})$ and light magenta $(\mathrm{Lm})$ inks in those regions. In order to predict the color of CMYLcLm prints, we make use of the fact that Lc and Lm have similar spectral characteristics as $\mathrm{C}$ and $\mathrm{M}$ respectively. The goal of this paper is to present a model to characterize a five-channel CMYLcLm printing system using a threechannel color prediction model, where we treat the ink combinations $\mathrm{Lc}+\mathrm{C}$ and $\mathrm{Lm}+\mathrm{M}$ as new compound inks. This characterization is based on our previous three-channel CMY color prediction model that is capable of predicting both colorimetric tri-stimulus values and spectral reflectance. The drawback of the proposed model in this paper is the requirement of large number of training samples. Strategies are proposed to reduce this number, which resulted in expected larger but acceptable color differences.
\end{abstract}

Keywords: Color prediction modeling, CMYLcLm printing, color difference, dot gain characterization

\section{INTRODUCTION}

In color printing the conventional CMYK (cyan, magenta, yellow and black) ink-set is extended to multi-channel printing by using additional inks, resulting in ink setups such as CMYKLcLm (adding light cyan-Lc, and light magentaLm), CMYKGO (adding green-G, and orange-O), CMYKRGB (adding red-R, green-G and blue-B), and other setups involving up to 12 channels. ${ }^{1}$ Introducing additional colorants is the result of the growing demand of high quality prints which require accurate color prediction models. Specifically, different black inks are used to prevent the substrates from being over inked and to produce finer gray shades. ${ }^{2}$ The introduction of Lc and Lm is usually aiming to reduce the graininess on the printed color images and the GO and RGB are applied to increase the saturation scope of the printable colors. ${ }^{3}$ However, applying additional inks has also brought up new challenges in the print device characterization. ${ }^{4}$ Color prediction models play an important role in this characterization and this paper is the result of our investigation on color prediction models for one of the above mentioned multi-channel printing setups, CMYLcLm.

To halftone the original continuous tone image, different shades on the image are represented by placing microdots with variation in their size, shape and/or frequency. On the binary halftoned image, to create the effect of a darker shade, the corresponding area is filled with larger and/or more densely placed microdots; while the lighter shades are reproduced by using fewer and/or sparse microdots. ${ }^{5}$ Therefore, in CMY printing, in the case of lighter shades, it may happen that the individual cyan (C) or magenta (M) dots are in a sparse pattern, standing out against the white background. ${ }^{6}$ These noticeable ink dots are undesirable in color printing. Therefore light cyan (Lc) and light magenta (Lm), which are lighter than $\mathrm{C}$ and $\mathrm{M}$ respectively, are applied in a denser pattern to alleviate the harshness of pure $\mathrm{C}$ and $\mathrm{M}$.

Figure 1 shows the spectra of full tone $\mathrm{C}$ and full tone Lc printed on uncoated paper using Canon image PROGRAF iPF6400 multichannel inkjet printer. The printed cyan patches were FM halftoned at 600dpi with varying reference coverage from 0 to $100 \%$ in step of $10 \%$, and were measured by a spectrophotometer BARBIERI electronic Spectro LFP RT, light source D65 with $2^{\circ}$ observer. Amongst these patches, the one with the closest spectrum to $100 \%$ Lc turned out to be the patch of $40 \%$ cyan, whose spectrum is also illustrated in Figure 1. This implies that reproducing a lighter cyan shade requires more $\mathrm{Lc}$ dots than $\mathrm{C}$ on the patch and thus with less chance to appear as visually unpleasant grainy ink dots. Thus, some printers may use halftone Lc or the mixture of halftone Lc and $\mathrm{C}$, instead of only halftone $\mathrm{C}$ to represent lighter cyan shades on the image. Similar strategy is applied by including Lm to remove the possible distinct and harsh M dots. This does not apply to low contrast yellow, which does not stand out harshly against white background.

*E-mail: paula.zitinski.elias@liu.se 


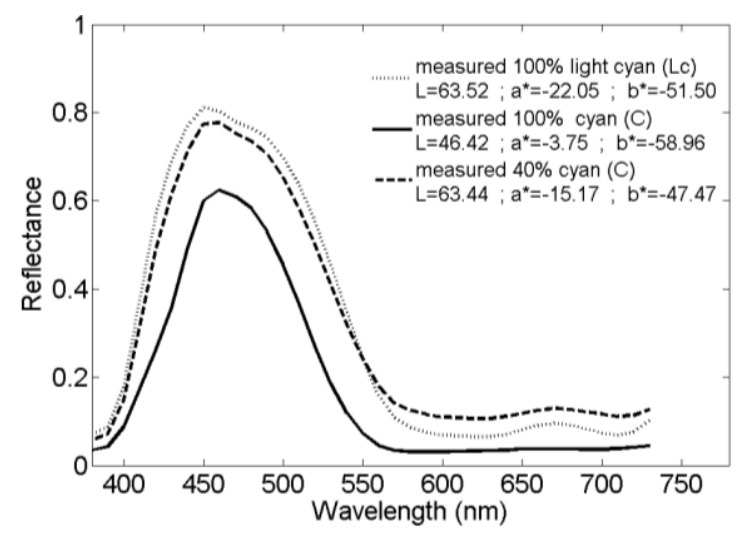

Figure 1. Measured spectra of full tone cyan (C), full tone light cyan (Lc) and halftone cyan (40\%).

There are several popular color prediction models such as the Yule-Nielsen Spectral modified Neugebauer (YNSN) model $^{7,8}$ and the Cellular Yule-Nielsen Spectral Neugebauer (CYNSN) model. ${ }^{9}$ Although these models were interpreted using the scenario of CMY printing, in theory they can be extended and used for more channels. For example the extension of Neugebauer-based models could be achieved by applying additional Neugebauer primaries resulted from the mixture of extra inks. The appendix of ref. 8 presents the extension of the Demichel equations ${ }^{10,11}$ from CMY to CMYK to figure out the fractional coverage of the 16 involved Neugebauer primaries.

In the case of halftone print involving extra inks such as GO (or RGB), Taplin and Berns ${ }^{12}$ applied the YNSN model to predict the color of prints using a CMYKGO inkjet printing system. Since six channels were involved, $64\left(2^{6}\right)$ Neugebauer primaries were required in the YNSN model. Also based on a CMYKGO inkjet printing system, Jang et al. ${ }^{13}$ estimated the colors after print by using the CYNSN model. In their investigation, the color samples were designed to avoid laying all inks simultaneously, in order to prevent ink blots. Thus the number of inks involved in each prediction was restricted to less than six.

Another strategy to predict the color of the prints of a multi-channel printing system was presented by Hung. ${ }^{14}$ It was proposed to subdivide the color gamut of the multi-channel printer into several sub-gamuts. Each sub-gamut includes only three or four ink coordinates. For example, the gamut built up by CMYKRGB printing is suggested to be subdivided into CMYK, CGYK, CBMK, MYRK, etc. Then the accuracy of the color prediction for CMYKRGB printing could be ensured by using well-developed color prediction models, which deal with only three or four ink coordinates in each sub-gamut.

For multi-channel printing with lighter colorants Lc and Lm (CMYKLcLm), Agar ${ }^{15}$ used the YNSN model involving 64 Neugebauer primaries to predict the colors of the CMYKLcLm prints. Son et al. ${ }^{16}$ also applied the YNSN model to predict the color of prints involving Lc and Lm. However, since in their research the concept of CMYKLcLm printing is to convert $\mathrm{C}$ or $\mathrm{M}$ to the combination of CLcLm and MYLm respectively, color prediction was carried out only for the MYLm and CLcLm prints.

Based on the above introduction about color prediction models for prints involving additional inks, we can conclude that two main strategies exist in literature. First, the extended popular color prediction models like the YNSN model and the CYNSN model are used. It must be noticed that the extended color prediction models may suffer from considerable computational requirements and inaccuracy compared to the case when only three inks are involved. ${ }^{16}$ Second, the printable colors are subdivided into sub-gamuts, each of which involves only three or four inks. The color prediction models for prints involving three or four inks are then used. This strategy ensures accurate color reproduction in multichannel printing but may result in color samples missing in the whole color gamut, especially in the darker region. ${ }^{13}$

The color prediction for CMYLcLm prints in this paper is based on our previous three-channel CMY color prediction model which is valid for predicting both colorimetric tri-stimulus values and spectral reflectance of the prints. A brief introduction of the three-channel CMY color prediction model is presented in the next section, followed by the explanation of the strategy that is proposed to extend this model to the CMYLcLm prints according to the properties of the added inks Lc and Lm. Experiments were carried out to evaluate the proposal and the corresponding results are illustrated and discussed in the final two sections in this paper. 


\section{COLOR PREDICTION MODEL FOR CMYLcLm PRINTING}

The mentioned color prediction model for CMY printing using an effective coverage map was presented in ref. 17. An effective coverage map is built using the measurement of several training CMY samples, based on CIEXYZ tri-stimulus values. This map held the effective coverage values of each ink correlated with certain reference ink combination. Then, given the reference coverage for cyan, magenta and yellow of any test patch, the effective coverage values of the involved inks could be estimated by cubic interpolation. Demichel's equations for three inks were used to estimate the fractional coverage for each primary ink (cyan, magenta and yellow), secondary ink (red, green, blue), black (mixture of CMY) and white (paper). As explained in ref. 17, the dot gain behavior of a certain ink varies at different wavelength bands, which was proved by characterizing the dot gain using CIEX, Y and Z stimulus respectively. To take into account this variation of dot gain behavior, the calculations in our model are based on CIEX, Y and Z stimulus in a similar manner. For simplicity, Equation (1) shows Demichel's equations using the CIEX values; similar formulations are applied using CIEY and $\mathrm{Z}$ values.

$$
\left\{\begin{array}{l}
c^{X}=c_{e f f}^{X} \cdot\left(1-m_{e f f}^{X}\right) \cdot\left(1-y_{e f f}^{X}\right) \\
m^{X}=m_{e f f}^{X} \cdot\left(1-c_{e f f}^{X}\right) \cdot\left(1-y_{e f f}^{X}\right) \\
y^{X}=y_{e f f}^{X} \cdot\left(1-m_{e f f}^{X}\right) \cdot\left(1-c_{e f f}^{X}\right) \\
r^{X}=m_{e f f}^{X} \cdot y_{e f f}^{X} \cdot\left(1-c_{e f f}^{X}\right) \\
g^{X}=c_{e f f}^{X} \cdot y_{e f f}^{X} \cdot\left(1-m_{e f f}^{X}\right) \\
b^{X}=c_{e f f}^{X} \cdot m_{e f f}^{X} \cdot\left(1-y_{e f f}^{X}\right) \\
k^{X}=c_{e f f}^{X} \cdot m_{e f f}^{X} \cdot y_{e f f}^{X} \\
p^{X}=\left(1-c_{e f f}^{X}\right) \cdot\left(1-m_{e f f}^{X}\right) \cdot\left(1-y_{e f f}^{X}\right)
\end{array}\right.
$$

$c_{\text {eff }}^{X}, m_{\text {eff }}^{X}$ and $y_{\text {eff }}^{X}$ are the estimated effective coverage values based on CIEX for cyan, magenta and yellow respectively, using the effective coverage map. $c^{X}, m^{X}, y^{X}, r^{X} g^{X}, b^{X}, k^{X}$ and $p^{X}$ are the fractional coverages for pure cyan, magenta, yellow, red, green, blue, black and paper respectively. Finally, the tri-stimulus values of the test CMY color print could be calculated using Equation (2).

$$
\left\{\begin{array}{l}
X_{c a l}=c^{X} \cdot X_{c}+m^{X} \cdot X_{m}+y^{X} \cdot X_{y}+r^{X} \cdot X_{r}+g^{X} \cdot X_{g}+b^{X} \cdot X_{b}+p^{X} \cdot X_{p}+k^{X} \cdot X_{k} \\
Y_{c a l}=c^{Y} \cdot Y_{c}+m^{Y} \cdot Y_{m}+y^{Y} \cdot Y_{y}+r^{Y} \cdot Y_{r}+g^{Y} \cdot Y_{g}+b^{Y} \cdot Y_{b}+p^{Y} \cdot Y_{p}+k^{Y} \cdot Y_{k} \\
Z_{c a l}=c^{Z} \cdot Z_{c}+m^{Z} \cdot Z_{m}+y^{Z} \cdot Z_{y}+r^{Z} \cdot Z_{r}+g^{Z} \cdot Z_{g}+b^{Z} \cdot Z_{b}+p^{Z} \cdot Z_{p}+k^{Z} \cdot Z_{k}
\end{array}\right.
$$

This model's accuracy for different printers and halftoning methods was verified by experiments in refs. 17 and 18 . The CIEX, Y and Z approximately represent three special wavelength bands along the visible wavelength interval. In ref. 19 , this model, based on CIEXYZ values, is extended to use spectral reflectance at different wavelength subintervals instead of CIEX, Y and $\mathrm{Z}$ values. The corresponding effective coverage map is built using spectral reflectance values, presenting the effective coverage values of each ink at different wavelength subintervals. Given any reference coverage, the effective coverage values of each ink are estimated by interpolation, and then used in Demichel's equations. Finally, the spectrum of the test color patch could be predicted by Equation (3).

$$
R_{c a l}^{i}=c^{i} \cdot R_{c}^{i}+m^{i} \cdot R_{m}^{i}+y^{i} \cdot R_{y}^{i}+r^{i} \cdot R_{r}^{i}+g^{i} \cdot R_{g}^{i}+b^{i} \cdot R_{b}^{i}+p^{i} \cdot R_{p}^{i}+k^{i} \cdot R_{k}^{i} \quad i=1,2,3, \ldots, 36
$$

Equation (3) uses the spectral reflectance at a certain wavelength subinterval ${ }^{19}$, the $i$-th subinterval, where $i=1,2,3, \ldots$, 36. $c^{i}, m^{i}, y^{i}, r^{i}, g^{i}, b^{i}, k^{i}$ and $p^{i}$ are the fractional coverages for cyan, magenta, yellow, red, green, blue, black and paper respectively. $R_{c}^{i}, R_{m}^{i}, R_{y}^{i}, R_{r}^{i}, R_{g}^{i}, R_{b}^{i}, R_{p}^{i}$ and $R_{k}^{i}$ are the $i$-th subinterval of the spectrum for each full tone primary colors, 
secondary colors, paper and black, respectively. $R_{\text {cal }}^{i}$ refers to the $i$-th subinterval of the predicted spectrum of the test patch.

The investigation on color prediction for CMYLcLm prints in the present paper is therefore carried out by involving more channels into the model established in refs. 17-20. As shown in Figure 1, the spectrum of full tone Lc shows a similar shape to that of full tone $\mathrm{C}$, although there is no linear correlation between the curves. Therefore we consider Lc to be associated with $\mathrm{C}$. Thus, Lc is not treated as a new, individual, ink when it is included together with $\mathrm{C}$. The same conclusion holds for Lm and $\mathrm{M}$ in our color prediction model for CMYLcLm prints.

Figure 2 shows the CIELAB values of the color patches produced by $\mathrm{M}$ and Lm in the CIELAB color space. The magenta curve connects the measured CIELAB values of $M$ patches with reference ink coverage increasing from 0 to $100 \%$ (by 10\% steps). The cyan curve is composed of CIELAB values of the Lm patches whose reference ink coverages also vary from 0 to $100 \%$ (by $10 \%$ steps). From the CIELAB values of these samples we can figure out that the $\mathrm{M}$ at $40 \%$ is the closest halftoned $\mathrm{M}$ patch to the full tone $\mathrm{Lm}$. These samples were printed using FM600dpi by the same Canon inkjet printer used in Section 1 and were measured by the same spectrophotometer. Figure 2 shows that in the CIELAB space although each halftone $M$ with reference coverage less than $40 \%$ has a certain halftone Lm patch located nearby, they cannot be replaced by any halftone Lm while preserving color accuracy. For example, in Figure 2, it is impossible to find a halftone Lm matching precisely the halftone patch with $20 \% \mathrm{M}$.

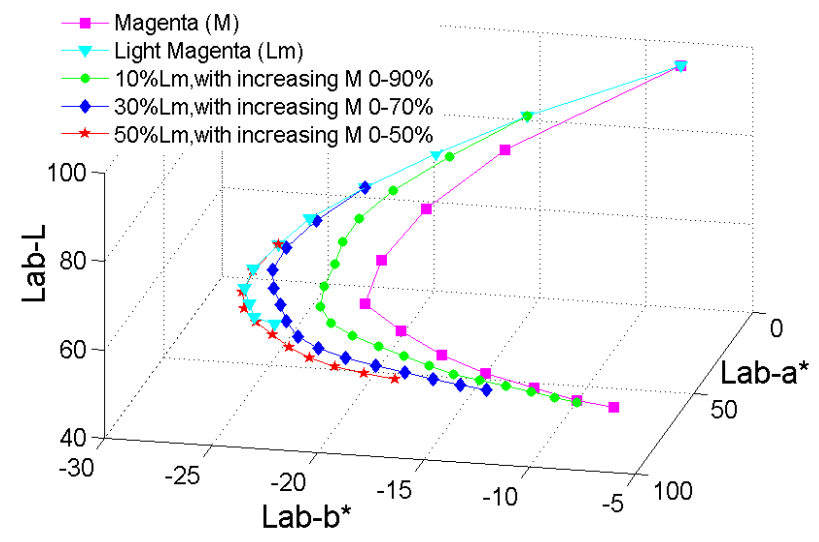

Figure 2. The colors of M+Lm patches shown in the CIELAB space.

Using our color prediction model for CMY printing, we predicted the CIELAB values of the patches of Lm printed together with M. As shown in Figure 2, the green, blue and red curves are the group of predicted colors of the patches containing both Lm and M. When producing a patch with darker shade, we considered that it is not necessary to have full tone $\mathrm{Lm}$ overlapping with full tone $\mathrm{M}$, i.e. we set the sum of reference coverages of $\mathrm{Lm}$ and $\mathrm{M}$ to less or equal to $100 \%$, hence Magenta $(\mathrm{M})+$ Light Magenta $(\mathrm{Lm}) \leq 1$. The samples on the green, blue and red curves in Figure 2 were designed by setting the reference coverage of $\mathrm{Lm}$ constant to $10 \%, 30 \%$ and $50 \%$ respectively while gradually increasing $\mathrm{M}$ from $0 \%$ until the total ink coverage reaches $100 \%$. These curves approximately represent the producible magenta shades with different dot saturation.

To study the color reproduction using both $\mathrm{Lm}$ and $\mathrm{M}$ together with other inks, we treat the mixture of $\mathrm{Lm}$ and $\mathrm{M}$ $(\mathrm{Lm}+\mathrm{M})$ as a compound ink, different from Lm or M. Similarly, the mixture of Lc and C (Lc+C) could be considered as another compound ink. Recall that three reference ink coordinates were used in our color prediction model. By treating $\mathrm{Lm}+\mathrm{M}(\mathrm{Lc}+\mathrm{C})$ as a compound ink to replace the $\mathrm{M}$ coordinate $(\mathrm{C}$ coordinate) in the model, we can apply our color prediction model without modification for the additional inks. Take the green curve in Figure 2 as example; it connects the samples having $\mathrm{Lm}+\mathrm{M}(\mathrm{Lm}=10 \%$ and $\mathrm{Lm}+\mathrm{M} \leq 1)$, which are numbered $\# 2$ to \#11 in Table 1 . The coverages of $\mathrm{Lm}$ and $\mathrm{M}$ on these samples are also presented in Table 1.

Table 1. The samples having $\mathrm{Lm}+\mathrm{M}(\mathrm{Lm}=10 \%$ and $\mathrm{Lm}+\mathrm{M} \leq 1)$

\begin{tabular}{|c|c|c|c|c|c|c|c|c|c|c|}
\hline Coverage (\%) & $\# 2$ & $\# 3$ & $\# 4$ & $\# 5$ & $\# 6$ & $\# 7$ & $\# 8$ & $\# 9$ & $\# 10$ & $\# 11$ \\
\hline Magenta (M) & 0 & 10 & 20 & 30 & 40 & 50 & 60 & 70 & 80 & 90 \\
\hline Light M (Lm) & 10 & 10 & 10 & 10 & 10 & 10 & 10 & 10 & 10 & 10 \\
\hline
\end{tabular}


To complete this curve with the patches that are lighter than \#2 or darker than \#11, the patch with bare paper and the one having heavier ink than \#11 are added and marked as \#1 and \#12 in Table 2. The samples in Table 2 are denoted by $\mathrm{Lm}+\mathrm{M}(\mathrm{Lm} \leq 10 \%, \mathrm{M} \in[0,0.1,0.2, \ldots, 0.9,1])$.

Table 2. $\mathrm{Lm}+\mathrm{M}(\mathrm{Lm} \leq 10 \%, \mathrm{M} \in[0,0.1,0.2, \ldots, 0.9,1])$, including two more patches

\begin{tabular}{|c|c|c|c|c|c|c|c|c|c|c|c|c|}
\hline Coverage (\%) & $\# 1$ & $\# 2$ & $\# 3$ & $\# 4$ & $\# 5$ & $\# 6$ & $\# 7$ & $\# 8$ & $\# 9$ & $\# 10$ & $\# 11$ & $\# 12$ \\
\hline Magenta (M) & 0 & 0 & 10 & 20 & 30 & 40 & 50 & 60 & 70 & 80 & 90 & 100 \\
\hline Light M (Lm) & 0 & 10 & 10 & 10 & 10 & 10 & 10 & 10 & 10 & 10 & 10 & 0 \\
\hline Normalized Lm+M & 0 & 4 & 14 & 24 & 34 & 44 & 54 & 64 & 74 & 84 & 94 & 100 \\
\hline
\end{tabular}

Since the mixture of $\mathrm{Lm}$ and $\mathrm{M}$ is considered a "new ink", the reference ink coverage of these Lm+M samples is normalized by: $M+\alpha \times \operatorname{Lm}$. We set $\alpha=0.4$ because the $40 \%$ halftone $M$ gives the closest CIELAB values to the full tone $\mathrm{Lm}$ according to the measurements. The normalized mixture $\mathrm{Lm}+\mathrm{M}(\mathrm{Lm} \leq 10 \%, \mathrm{M} \in[0,0.1,0.2, \ldots, 0.9,1])$ is represented in the last row in Table 2. Similarly, the combinations $\mathrm{Lm}+\mathrm{M}(\mathrm{Lm} \leq 30 \%)$ on the blue curve in Figure 2 are completed and are denoted by $\mathrm{Lm}+\mathrm{M}(\mathrm{Lm} \leq 30 \%, \mathrm{M} \in[0,0.1,0.2, \ldots, 0.9,1])$. Using the same strategy, several groups of $\mathrm{Lm}+\mathrm{M}$ mixtures are applied and therefore several "new inks" associated with magenta are "created". For Lc+C, similar processes are carried out. The $\mathrm{C}$ and $\mathrm{M}$ ink coordinates in our model are replaced by the new ones, which are the different mixtures of $\mathrm{Lc}+\mathrm{C}$ and $\mathrm{Lm}+\mathrm{M}$ respectively.

According to the principle of the effective coverage map in our model, several training samples are required to build up the map. The more compound ink introduced, the more training samples are required. In order to avoid using too many training samples, we only use $\mathrm{Lm}+\mathrm{M}(\mathrm{Lm} \leq$ area, area $\in[0,0.13,0.33,0.66,1])$ instead of $\mathrm{Lm}+\mathrm{M}(\mathrm{Lm} \leq$ area, area $\in[0$, $0.1,0.2, \ldots, 0.9,1])$ that are used for interpretation in Table 2 . The reason for choosing ink coverages $[0,0.13,0.33,0.66$, 1] instead of e.g. $[0,0.25,0.5,0.75,1]$ is explained in ref. 18: the characterization curves for magenta were not symmetrical around 0.5 and the reference coverage which produced the biggest dot gain was shifted to the left and is located around 0.33 . The similar situation happens for both cyan and yellow, thus, we use $\mathrm{Lc}+\mathrm{C}$ ( $\mathrm{Lc} \leq$ area, area $\in[0$, $0.13,0.33,0.66,1])$ for the training samples involving $L c$ and $C$, and $Y \in[0,0.13,0.33,0.66,1]$ for the training samples involving Y. Figure 3 illustrates the workflow for choosing the mixture group $\mathrm{Lm}+\mathrm{M}(\mathrm{Lm} \leq 0.13, \mathrm{M} \in[0,0.13,0.33$, $0.66,1])$, which is considered as a new compound ink in the model.

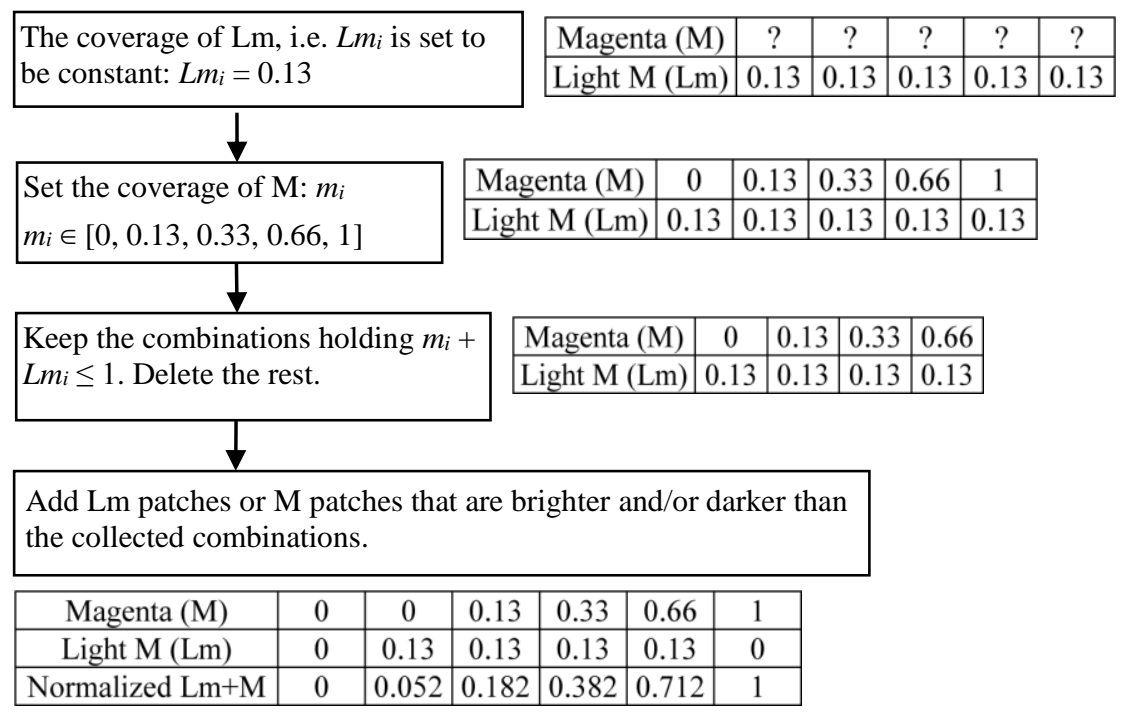

Figure 3. Creating mixture group $\mathrm{Lm}+\mathrm{M}(\mathrm{Lm} \leq 13 \%, \mathrm{M} \in[0,0.13,0.33,0.66,1])$.

Different groups of $\mathrm{Lm}+\mathrm{M}$ compound inks are the result of using the workflow explained in Figure $3 ; \mathrm{Lm}+\mathrm{M}$ (Lm $\leq$ $0.13 ; \mathrm{Lm} \leq 0.33 ; \mathrm{Lm} \leq 0.66, \mathrm{M} \in[0,0.13,0.33,0.66,1])$. Table 3 shows the example of mixing $\mathrm{Lm}+\mathrm{M}$ inks $(\mathrm{Lm} \leq 0.66$, $\mathrm{M} \in[0,0.13,0.33,0.66,1])$. 
Table 3. Mixture group $\mathrm{Lm}+\mathrm{M}(\mathrm{Lm} \leq 66 \%, \mathrm{M} \in[0,0.13,0.33,0.66,1])$.

\begin{tabular}{|c|c|c|c|c|c|c|c|}
\hline Magenta (M) & 0 & 0 & 0 & 0 & 0.13 & 0.33 & 1 \\
\hline Light M (Lm) & 0 & 0.13 & 0.33 & 0.66 & 0.66 & 0.66 & 0 \\
\hline Normalized Lm+M & 0 & 0.052 & 0.132 & 0.264 & 0.394 & 0.594 & 1 \\
\hline
\end{tabular}

The individual ink $\mathrm{Lm}$ is also included and is a specific mixture group: $\mathrm{Lm}+\mathrm{M}(\mathrm{Lm} \leq 1, \mathrm{M} \in[0,0.13,0.33,0.66,1])$, as shown in Table 4. In Table 4 the normalized reference coverages of $\mathrm{Lm}+\mathrm{M}$ are the coverage values of $\mathrm{Lm}$, instead of normalization $\mathrm{M}+\alpha \times \mathrm{Lm}$. The reason is that if only $\mathrm{Lm}$ is involved, it is treated as an individual ink coordinate replacing the coordinate $\mathrm{M}$ in the CMY color prediction models.

Table 4. Mixture group $\mathrm{Lm}+\mathrm{M}(\mathrm{Lm} \leq 1, \mathrm{M} \in[0,0.13,0.33,0.66,1])$.

\begin{tabular}{|c|c|c|c|c|c|}
\hline Magenta (M) & 0 & 0 & 0 & 0 & 0 \\
\hline Light M (Lm) & 0 & 0.13 & 0.33 & 0.66 & 1 \\
\hline Normalized Lm+M & 0 & 0.13 & 0.33 & 0.66 & 1 \\
\hline
\end{tabular}

To uniform the appellation, the mixtures of $\mathrm{Lm}$ and $\mathrm{M}$ are generally denoted by $\mathrm{Lm}+\mathrm{M}$ (Lm $\leq$ area, area $\in[0,0.13,0.33$, $0.66,1] ; \mathrm{M} \in[0,0.13,0.33,0.66,1])$. To explain how to replace $\mathrm{C}$ and $\mathrm{M}$ with $\mathrm{Lc}+\mathrm{C}$ and $\mathrm{Lm}+\mathrm{M}$ respectively the 2-D effective coverage grid, proposed and explained in ref. 17, for the training samples is used, as shown in Figure 4. Lc+C $(\mathrm{Lc} \leq 0.33, \mathrm{C} \in[0,0.13,0.33,0.66,1])$ and $\mathrm{Lm}+\mathrm{M}(\mathrm{Lm} \leq 0.13, \mathrm{M} \in[0,0.13,0.33,0.66,1])$ are the ink coordinates in Figure 4, representing the previous coordinates $\mathrm{C}$ and $\mathrm{M}$.

All the required training samples with $\mathrm{Lc}+\mathrm{C}(\mathrm{Lc} \leq 0.33, \mathrm{C} \in[0,0.13,0.33,0.66,1])$ and $\mathrm{Lm}+\mathrm{M}(\mathrm{Lm} \leq 0.13, \mathrm{M} \in[0$, $0.13,0.33,0.66,1])$ are presented in Figure 4 . Recall that $\mathrm{Lc}+\mathrm{C}$ and $\mathrm{Lm}+\mathrm{M}$ are treated as individual ink coordinates like $\mathrm{C}$ and $\mathrm{M}$ were treated in our model for CMY printing. As soon as the measured data (CIEXYZ tri-stimulus values or spectral reflectance) of the training samples in Figure 4 are obtained, the grid would be filled with effective coverage values by mapping.

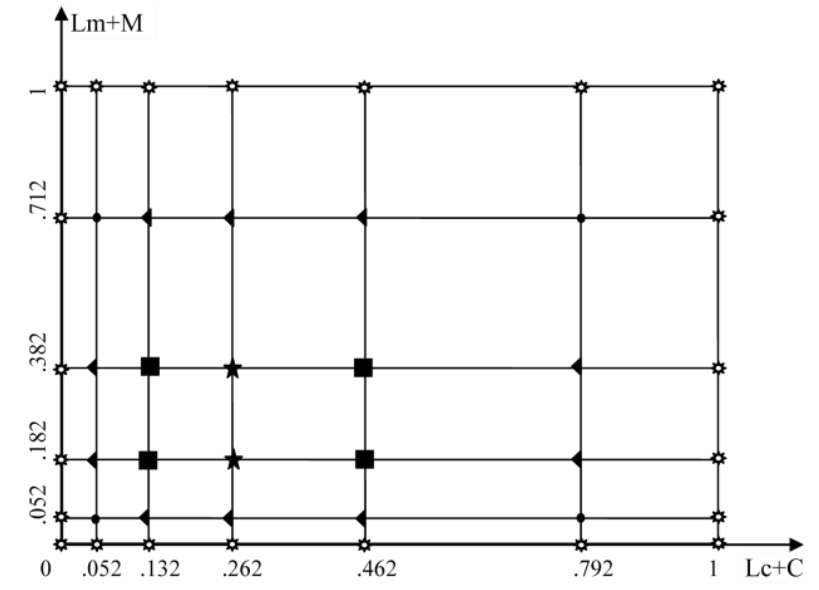

Figure 4. The $7 \times 6$ effective coverage grid for the training samples using $\mathrm{Lc}+\mathrm{C}$ and $\mathrm{Lm}+\mathrm{M}$.

$\mathrm{Lm}+\mathrm{M}(\mathrm{Lm} \leq$ area, area $\in[0,0.13,0.33,0.66,1] ; \mathrm{M} \in[0,0.13,0.33,0.66,1])$ includes five groups of $\mathrm{Lm}+\mathrm{M}$ combinations. The same is valid for $\mathrm{Lc}+\mathrm{C}$. Therefore $5 \times 5=25$ effective coverage grids would be created to predict the color of any CMLcLm prints, one of which is shown in Figure 4. Given the reference coverage of a certain CMLcLm print, one grid is selected from the 25 grids according to the reference coverage. For instance, the reference coverage of a CMLcLm patch given as $(0.22,0.4,0.1,0.5)$ means that the reference coverage of $\mathrm{Lc}(0.1)$ is closer to 0.13 out of $[0$, $0.13,0.33,0.66,1]$ and the reference of $\operatorname{Lm}(0.5)$ is closer to 0.66 out of $[0,0.13,0.33,0.66,1]$. Therefore the grid that was created by using ink coordinates $\mathrm{Lc}+\mathrm{C}(\mathrm{Lc} \leq 0.13, \mathrm{C} \in[0,0.13,0.33,0.66,1])$ and $\mathrm{Lm}+\mathrm{M}(\mathrm{Lm} \leq 0.66, \mathrm{M} \in[0,0.13$, $0.33,0.66,1])$ would be chosen to determine the effective coverage values for this patch. 
For CMLcLm prints, the involved primaries in our model are on the four corners of the chosen grid. For example, if the grid shown in Figure 4 was chosen for a given test CMLcLm print, the used primaries are the full tone $\mathrm{C}$, full tone $\mathrm{M}$, full tone $\mathrm{C}$ mixed with full tone $\mathrm{M}$ and bare paper. As mentioned above, it is assumed that $\mathrm{Lc}+\mathrm{C} \leq 1$ and $\mathrm{Lm}+\mathrm{M} \leq 1$ for the prints in this paper. After the effective coverage values are obtained by cubic interpolation through the chosen grid, the color of the test CMLcLm sample will be predicted by Equations (2) based on CIEX, Y and Z values or by Equation (3) based on spectral reflectance.

The effective coverage grids involving ink coordinates [Lc+C and $\mathrm{Y}]$ or $[\mathrm{Lm}+\mathrm{M}$ and $\mathrm{Y}]$ could be obtained similarly by using the measurements of the corresponding training samples. The color of the test CYLc or MYLm samples could then be predicted similar to those of CMLcLm test samples.

When predicting the test samples containing all the channels, i.e. CMYLcLm, notice again that $\mathrm{Lc}+\mathrm{C}$ and $\mathrm{Lm}+\mathrm{M}$ are treated as compound inks replacing the $\mathrm{C}$ and $\mathrm{M}$ ink coordinates in our previous model. The effective coverage maps for $(\mathrm{Lc}+\mathrm{C})(\mathrm{Lm}+\mathrm{M}) \mathrm{Y}$ are built up by the same processing that was explained for CMY in ref. 17. The required training samples are prepared similarly to those for the effective coverage grid shown in Figure 4. For example, for the ink coordinates:

$\mathrm{Lc}+\mathrm{C}: \mathrm{Lc}+\mathrm{C}(\mathrm{Lc} \leq 0.33, \mathrm{C} \in[0,0.13,0.33,0.66,1])$

$\mathrm{Lm}+\mathrm{M}: \mathrm{Lm}+\mathrm{M}(\mathrm{Lm} \leq 0.13, \mathrm{M} \in[0,0.13,0.33,0.66,1])$

$\mathrm{Y}: \mathrm{Y} \in[0,0.13,0.33,0.66,1]$

the effective coverage map is created by the measurements of the training samples with the reference ink components listed in Table 5.

Table 5. The reference ink coordinates of the training samples for certain effective coverage map by $(\mathrm{Lc}+\mathrm{C})(\mathrm{Lm}+\mathrm{M}) \mathrm{Y}$

\begin{tabular}{|c|c|c|c|c|c|c|c|}
\hline Cyan (C) & 0 & 0 & 0 & 0.13 & 0.33 & 0.66 & 1 \\
\hline Light C (Lc) & 0 & 0.13 & 0.33 & 0.33 & 0.33 & 0.33 & 0 \\
\hline Normalized Lc+C & 0 & 0.052 & 0.132 & 0.262 & 0.462 & 0.792 & 1 \\
\hline Magenta (M) & 0 & 0 & 0.13 & 0.33 & 0.66 & 1 & \\
\hline Light M (Lm) & 0 & 0.13 & 0.13 & 0.13 & 0.13 & 0 & \\
\hline Normalized Lm+M & 0 & 0.052 & 0.182 & 0.382 & 0.712 & 1 & \\
\hline Yellow (Y) & 0 & 0.13 & 0.33 & 0.66 & 1 & & \\
\hline
\end{tabular}

Therefore totally $7 \times 6 \times 5=210$ training samples are involved in this chosen effective coverage map. These training samples actually include the training ones that were used for the effective coverage grid in Figure 4. Given any reference CMYLcLm combination, the effective coverage values for the primaries are obtained by cubic interpolation through the selected effective coverage map, which is chosen in advance according to the reference (Lc+C), $(\mathrm{Lm}+\mathrm{M})$ and $\mathrm{Y}$. Finally, the color of the test CMYLcLm print is predicted using Equation (2) based on CIEXYZ tri-stimulus values or by Equation (3) based on spectral reflectance.

\section{EXPERIMENTS AND DISCUSSION}

To evaluate the approach introduced in Section 2, a large number of CMYLcLm prints were printed and measured. The used printer was the Canon imagePROGRAF iPF6400 multichannel inkjet printer. Uncoated paper for ink jet color printing was used. FM600dpi was used to create the halftone patches. All the prints were then measured by the spectrophotometer BARBIERI electronic Spectro LFP RT, light source D65 with $2^{\circ}$ observer.

The prints include training samples and test samples. The reference coverages $\mathrm{Lc}+\mathrm{C} \leq 1$ and $\mathrm{Lm}+\mathrm{M} \leq 1 \mathrm{during}$ the design of the test samples were assumed. The print group CMLcLm refers to the samples containing C, M, Lc and Lm. Print groups CYLc and MYLm are also defined according to the ink combinations of the test samples. Furthermore, there were also groups such as CMYLmLc, CYLmLc, CMYLc, MYLmLc and CMYLm.

For a better evaluation, the color prediction for test samples in groups CMLcLm, CYLc and MYLm were carried out apart from the ones in groups CMYLmLc, CYLmLc, CMYLc, MYLmLc and CMYLm. The reason is that in order to predict the colors of the prints in groups CMLcLm (596 test samples), CYLc (368 test samples) and MYLm (368 test 
samples), only the effective coverage grids (two dimensional) were needed, while for the print groups CMYLcLm etc. the full effective coverage maps (three dimensional) were required. Regarding the reference ink coverages of the test samples in these groups, half of the samples only used values such as $0.25,0.5$ and 0.75 for the involved inks and the rest of the test samples had random reference ink combinations. To simplify the experiment, during the color prediction using effective coverage maps, the prints in groups CYLmLc etc were all classified into group CMYLcLm (534 random test samples). All the test samples used in this experiment excluded the training samples introduced before.

\subsection{CMLcLm, CYLc, MYLm}

By considering $(\mathrm{Lc}+\mathrm{C})$ and $(\mathrm{Lm}+\mathrm{M})$ as two individual ink coordinates in the color prediction model, the prediction accuracy of our model for the prints in groups CMLcLm, CYLc, MYLm is presented in Table 6, using $\Delta \mathrm{E}_{94}$ color differences and spectral $\triangle \mathrm{RMS}$.

Table 6. The color difference between measured and predicted colors

The designed reference coverage of the training samples for the effective coverage grids:
$L c+C(L c \leq$ area, area $\in[0,0.13,0.33,0.66,1] ; C \in[0,0.13,0.33,0.66,1]) ;$
$L m+M(L m \leq$ area, area $\in[0,0.13,0.33,0.66,1] ; M \in[0,0.13,0.33,0.66,1]) ;$
$Y \in[0,0.13,0.33,0.66,1]$

Based on CIEXYZ

\begin{tabular}{|c|c|c|c|}
\hline$\Delta E_{94}$ & $\begin{array}{c}\text { CMLcLm } \\
\text { (596 samples) }\end{array}$ & $\begin{array}{c}\text { CYLc } \\
\text { (368 samples) }\end{array}$ & $\begin{array}{c}\text { MYLm } \\
\text { (368 samples) }\end{array}$ \\
\hline $\operatorname{Max}$ & 3.48 & 3.90 & 2.82 \\
\hline mean & 0.58 & 0.95 & 0.69 \\
\hline$>4$ & 0 & 0 & 0 \\
\hline \multicolumn{4}{|c|}{ Based on Spectral Reflectance } \\
\hline$\triangle R M S$ & $\begin{array}{c}\text { CMLcLm } \\
\text { (596 samples) }\end{array}$ & $\begin{array}{c}\text { CYLc } \\
\text { (368 samples) }\end{array}$ & $\begin{array}{c}\text { MYLm } \\
\text { (368 samples) }\end{array}$ \\
\hline $\operatorname{Max}$ & 0.0419 & 0.0222 & 0.0240 \\
\hline mean & 0.0088 & 0.0065 & 0.0067 \\
\hline
\end{tabular}

The mean prediction errors shown in Table 6 based on CIEXYZ values for the groups CMLcLm, CYLc, MYLm are small. It is hard to evaluate the performance of the prediction based on spectral reflectance by the obtained maximum spectral $\triangle$ RMS, which is 0.0419 in Table 6. However, considering that the obtained spectral $\Delta$ RMS between two white patches (two blank patches at different positions on the print sheet) was 0.0065 , we believe that the obtained mean spectral $\triangle$ RMSs $(0.0088,0.0065$ and 0.0067$)$ in Table 6 are acceptable.

The number of the training samples used in this experiment is as follows. Different mixtures of Lm+M were applied as ink coordinates in different effective coverage grids. As explained in the introduction in Section 2, five grids are created for CMLcLm prints, and the size of each grid varies. However, most of the Lm+M combinations are taken in different grids repeatedly. According to our experiment, a total of 17 combinations of $\mathrm{Lm}+\mathrm{M}$ are required by setting $\mathrm{Lm}+\mathrm{M}$ ( $\mathrm{Lm}$ $\leq$ area, area $\in[0,0.13,0.33,0.66,1] ; \mathrm{M} \in[0,0.13,0.33,0.66,1])$. Similarly, $17 \mathrm{Lc}+\mathrm{C}$ combinations are required by setting $\mathrm{Lc}+\mathrm{C}(\mathrm{Lc} \leq$ area, area $\in[0,0.13,0.33,0.66,1] ; \mathrm{C} \in[0,0.13,0.33,0.66,1])$. Therefore $17 \times 17=289$ training samples are designed for the CMLcLm prints and $17 \times 5=85$ training samples are designed for the CYLc or MYLm prints.

To cut down the number of the training samples, we applied the setting $\mathrm{Lm}+\mathrm{M}(\mathrm{Lm} \leq$ area, area $\in[0,0.33,0.66,1] ; \mathrm{M} \in$ $[0,0.33,0.66,1])$ and $\mathrm{Lc}+\mathrm{C}(\mathrm{Lc} \leq$ area, area $\in[0,0.33,0.66,1] ; \mathrm{C} \in[0,0.33,0.66,1])$. The required number of training samples lowers down to $10 \times 10=100$ for CMLcLm prints and $10 \times 5=50$ for the CYLc or MYLm prints. The corresponding prediction errors are presented in Table 7.

As expected, the errors in Table 7 are larger than those in Table 6, especially for the print groups CYLc and MYLm. We believe it is possible to improve the performance of the model by applying more suitable reference combinations for the training samples. Until present date, we tried the setting $\mathrm{Lm}+\mathrm{M}(\mathrm{Lm} \leq$ area, area $\in[0,0.33,0.66,1] ; \mathrm{M} \in[0,0.33,0.66$, 1]) and $\mathrm{Lc}+\mathrm{C}(\mathrm{Lc} \leq$ area, area $\in[0,0.33,0.66,1] ; \mathrm{C} \in[0,0.33,0.66,1])$, but it might be possible to decrease the prediction error with fewer training samples by using other settings such as $\mathrm{Lm}+\mathrm{M}(\mathrm{Lm} \leq$ area, area $\in[0,0.33,1] ; \mathrm{M} \in$ $[0,0.33,0.66,1]), \mathrm{Lc}+\mathrm{C}(\mathrm{Lc} \leq$ area, area $\in[0,0.33,1] ; \mathrm{C} \in[0,0.25,0.66,1])$ and $\mathrm{Y}(\mathrm{Y} \in[0,0.25,0.66,1])$. 
It has to be noticed that the way to normalize the reference coverage of the mixture $\mathrm{Lm}+\mathrm{M}$ or $\mathrm{Lc}+\mathrm{C}$ may affect the performance of the color prediction model. The normalized reference coverages are used in the cubic interpolation through the effective coverage maps to calculate the effective coverage values for each Neugebauer primary. Recall that we used $\mathrm{M}+\alpha \times \mathrm{Lm}, \alpha=0.4$ for $\mathrm{Lm}+\mathrm{M}$ considering the $40 \%$ halftone $\mathrm{M}$ gives the closest CIELAB values to the full tone $\mathrm{Lm}$. $\mathrm{C}+\alpha \times \mathrm{Lc}$ with $\alpha=0.4$ was used for $\mathrm{Lc}+\mathrm{C}$ for the same reason. In the future, it is of interest to apply varying $\alpha$ for Lc and $\mathrm{Lm}$ with different reference coverages during the reference coverage normalization.

Table 7. The color difference between measured and predicted colors, using less training samples

\begin{tabular}{|c|c|c|c|}
\hline \multicolumn{4}{|c|}{$\begin{array}{l}\text { The designed reference coverage of the training samples for the effect } \\
\text { grids: } \\
L c+C(L c \leq \text { area, area } \in[0,0.33,0.66,1] ; C \in[0,0.33,0.66,1]) ; \\
L m+M(L m \leq \text { area, area } \in[0,0.33,0.66,1] ; M \in[0,0.33,0.66,1]) \text {; } \\
Y \in[0,0.13,0.33,0.66,1]\end{array}$} \\
\hline \multicolumn{4}{|c|}{ Based on CIEXYZ } \\
\hline$\Delta E_{94}$ & $\begin{array}{c}\text { CMLcLm } \\
\text { (596 samples) }\end{array}$ & $\begin{array}{c}\text { CYLc } \\
\text { (368 samples) }\end{array}$ & $\begin{array}{c}\text { MYLm } \\
\text { (368 samples) }\end{array}$ \\
\hline Max & 4.97 & $6.58^{1}$ & 6.78 \\
\hline mean & 1.38 & 1.61 & 1.17 \\
\hline$>4$ & 6 & 16 & 9 \\
\hline \multicolumn{4}{|c|}{ Based on Spectral Reflectance } \\
\hline$\triangle R M S$ & $\begin{array}{c}\text { CMLcLm } \\
\text { (596 samples) }\end{array}$ & $\begin{array}{c}\text { CYLc } \\
\text { (368 samples) }\end{array}$ & $\begin{array}{c}\text { MYLm } \\
\text { (368 samples) }\end{array}$ \\
\hline $\begin{array}{l}\text { Max } \\
\text { mean }\end{array}$ & $\begin{array}{l}0.0863 \\
0.0191\end{array}$ & $\begin{array}{l}0.0788 \\
0.0129\end{array}$ & $\begin{array}{l}0.0373 \\
0.0089\end{array}$ \\
\hline
\end{tabular}

\subsection{CMYLcLm}

For the prints in group CMYLmLc, to find out the effective coverage values for the involved Neugebauer primaries, the effective coverage maps are needed. As mentioned in Section 2, if we choose the setting $\mathrm{Lc}+\mathrm{C}$ ( $\mathrm{Lc} \leq$ area, area $\in[0,0.13$, $0.33,0.66,1] ; \mathrm{C} \in[0,0.13,0.33,0.66,1])$, for the training samples, $17 \mathrm{Lc}+\mathrm{C}$ combinations were required, as shown in Table 8 . The same combinations are required for $\mathrm{Lm}+\mathrm{M}$, which were rearranged into five groups. Since $\mathrm{Y} \in[0,0.13$, $0.33,0.66,1]$, totally 25 effective coverage maps with different sizes were built up. The total number of used training samples was $17 \times 17 \times 5=1445$ (all the training samples used in the effective coverage grids were also included). We have to admit that the requirement of such number of training samples lowers the advantage of this prediction model.

Table 8. All the required $\mathrm{Lc}+\mathrm{C}$ for the $\mathrm{Lc}+\mathrm{C}(\mathrm{Lc} \leq$ area, area $\in[0,0.13,0.33,0.66,1] ; \mathrm{C} \in[0,0.13,0.33,0.66,1])$

\begin{tabular}{|c|c|c|c|c|c|c|c|c|c|}
\hline No. & $\# 1$ & $\# 2$ & $\# 3$ & $\# 4$ & $\# 5$ & $\# 6$ & $\# 7$ & $\# 8$ & $\# 9$ \\
\hline Cyan $(\mathrm{C})$ & 0 & 0 & 0.13 & 0 & 0.13 & 0.13 & 0 & 0.33 & 0.33 \\
\hline Light C $(\mathrm{Lc})$ & 0 & 0.13 & 0 & 0.33 & 0.13 & 0.33 & 0.66 & 0 & 0.13 \\
\hline$\alpha \times$ Lc $+\mathrm{C}, \alpha=0.4$ & 0 & 0.052 & 0.13 & 0.132 & 0.182 & 0.262 & 0.264 & 0.33 & 0.382 \\
\hline No. & $\# 10$ & $\# 11$ & $\# 12$ & $\# 13$ & $\# 14$ & $\# 15$ & $\# 16$ & $\# 17$ & \\
\hline Cyan $(\mathrm{C})$ & 0.13 & 0 & 0.33 & 0.33 & 0.66 & 0.66 & 0.66 & 1 & \\
\hline Light C $(\mathrm{Lc})$ & 0.66 & 1 & 0.33 & 0.66 & 0 & 0.13 & 0.33 & 0 & \\
\hline$\alpha \times$ Lc+C,$\alpha=0.4$ & 0.394 & 0.4 & 0.462 & 0.594 & 0.66 & 0.712 & 0.792 & 1 & \\
\hline
\end{tabular}

To cut down the number of required training samples, we attempted to create a single effective coverage map with larger size to substitute the 25 effective coverage maps with different sizes. The map with $17 \times 17 \times 5$ elements is too large and therefore we attempted removing several ink combinations from Table 8 . The combinations for Lc+C marked with gray in Table 8 are the ones chosen to be removed. These removed combinations include the ones having only one ink (\#2, \#3, $\# 4$, \#7, \#8, \#11, \#14), since the aimed effective coverage map was created mainly for the test samples in group CMYLmLc, and the one (\#10) having a close normalized reference coverage to its neighboring combination (\#9). Finally, nine combinations were kept for Lc+C, as shown in Table 9. 
Table 9. The kept combinations for $\mathrm{Lc}+\mathrm{C}$

\begin{tabular}{|c|c|c|c|c|c|c|c|c|c|}
\hline New No. & $\# 1$ & $\# 2$ & $\# 3$ & $\# 4$ & $\# 5$ & $\# 6$ & $\# 7$ & $\# 8$ & $\# 9$ \\
\hline Cyan $(\mathrm{C})$ & 0 & 0.13 & 0.13 & 0.33 & 0.33 & 0.33 & 0.66 & 0.66 & 1 \\
\hline Light C $(\mathrm{Lc})$ & 0 & 0.13 & 0.33 & 0.13 & 0.33 & 0.66 & 0.13 & 0.33 & 0 \\
\hline$\alpha \times$ Lc $+\mathrm{C}, \alpha=0.4$ & 0 & 0.182 & 0.262 & 0.382 & 0.462 & 0.594 & 0.712 & 0.792 & 1 \\
\hline
\end{tabular}

The same nine combinations were kept for $\mathrm{Lm}+\mathrm{M}$, leading to $9 \times 9 \times 5=405$ training samples in one effective coverage map. The created effective coverage map was used for 534 random test samples in group CMYLmLc. So far, only the color prediction based on CIEXYZ tri-stimulus values has been carried out using this $9 \times 9 \times 5$ effective coverage map. Figure 5 shows the distribution of the obtained $\Delta \mathrm{E}_{94}$ color differences between the predicted and measured colors.

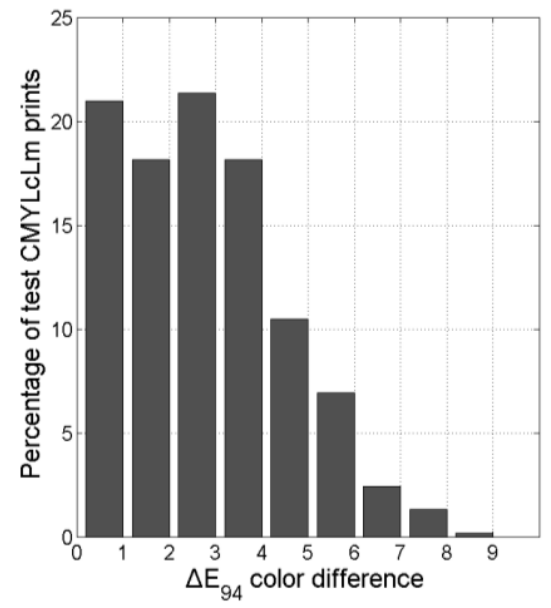

Figure 5. Distribution of the $\Delta \mathrm{E}_{94}$ between predicted and measured colors.

As shown in Figure 5, approximately $78 \%(21+18+21+18)$ of the test samples the $\Delta \mathrm{E}_{94}$ color differences are less than 4 while for around 4 percent of the test samples the $\Delta \mathrm{E}_{94}$ color differences are over 6 . The mean value of the obtained $\Delta \mathrm{E}_{94}$ color differences is 2.18 while the maximum value is 8.32 . This prediction accuracy for the CMYLmLc prints is not as satisfying as expected before the experiment. In future work, it is suggested to consider the fact that in practical CMYLmLc printing, it may be rare to use the five inks simultaneously with heavy reference coverage, such as CMYLmLc $=(45 \%, 45 \%, 35 \%, 45 \%, 50 \%)$.

\section{CONCLUSIONS}

This paper presents our investigation on the color prediction model for five-channel CMYLmLc printing. Unlike the Yule-Nielsen Spectral modified Neugebauer (YNSN) model or the Cellular Yule-Nielsen Spectral Neugebauer (CYNSN) model, we treated the ink combination $\mathrm{C}$ and $\mathrm{Lc}$ and the ink combination $\mathrm{M}$ and Lm, as new, compound inks, replacing $\mathrm{C}$ and $\mathrm{M}$ in our previous three-channel color prediction model. Computation based on CIEXYZ values and spectral reflectances were carried out to evaluate the proposed model. The color prediction for prints in groups CMLcLm, CYLc and MYLm were good and satisfying. Strategies for reducing the training samples were applied while giving acceptable prediction accuracy. The modification of the model for print group CMYLmLc is a part of our future study to evaluate and improve our color prediction model. We believe that the investigation presented in this paper is valuable for the color separation and thereby for the characterization of printing systems. 


\section{REFERENCES}

[1] Steinmueller, U. and Gulbins, J., [The Art of Fine Art Printing: Using today's Inkjet Printers for Quality Prints], Steinmuller Photo (2006).

[2] Pring R., [Www.Color], Watson-Guptill (2000).

[3] Jang I., Son Ch., Park T., Ko K. and Ha Y., "Colorimetric characterization based on color correlation in CMYKGO printer," Proc. SPIE-IS\&T Electronic Imaging, Color Imaging, 6058, 60580p1-p10 (2005).

[4] Zeng H., "3-D color separation maximizing the printer gamut," Proc. SPIE-IS\&T Electronic Imaging, Color Imaging, 5008, 260-267 (2003).

[5] Gooran, S., "Dependent Color Halftoning, Better Quality with Less Ink," J. Imaging Sci. Technol., 48(4), 354362 (2004).

[6] Son Ch., Kim Y., Lee Ch., and Ha Y., "Six color separation for improving graininess in a middle tone region," Proc. SPIE and IS\&T, Color Imaging IX: Processing, Hardcopy, and Applications, 110-120 (2004).

[7] Viggiano J. A. S., "Modeling the Color of Multi-Colored Halftones," Proc. TAGA, 44-62 (1990).

[8] Hersch, R.D. and Crété, F., "Improving the Yule-Nielsen modified spectral Neugebauer model by dot surface coverages depending on the ink superposition conditions," Proc. IS\&T/SPIE Electronic Imaging Symposium 5667, 434-445 (2005).

[9] Chen Y., Berns R., and Taplin L., "Six color printer characterization using an optimized cellular Yule-Nielsen spectral Neugebauer model," J. Imaging Sci. Technol. 48, 519-528 (2004).

[10] Demichel, M., Le Procédé, 26, 17-21, 26-27 (1924).

[11] Rogers, G. L., " Neugebauer revisited: random dots in halftone screening," Color Research \& Application, 23(2), $104-113$ (1998).

[12] Taplin L. and Berns R., "Spectral Color Reproduction Based on Six-Color Inkjet Output System," Proc. 9th Color Imaging Conference: Color Science and Engineering: Systems, Technologies, and Applications Scottsdale, 209-213 (2001).

[13] Jang I., Son Ch., Park T., Ko K. and Ha Y., "Hi-Fi Printer Characterization Method using Color Correlation for Gamut Extension," Image Processing, 2006 IEEE International Conference on, (2006).

[14]Hung P. C., "Colorimetric characterization beyond three colorants," Proc. SPIE Color Imaging; DeviceIndependent Color, Color Hardcopy, and Graphic Arts, San Jose, 3963, 196-207 (2000).

[15] Agar U., "Model Based Color Separation for CMYKcm Printing," Proc. 9th Color Imaging Conference (2001).

[16] Son C., Park H. and Ha Y., "Improved Color Separation Based on Dot-Visibility Modeling and Color Mixing Rule for Six-Color Printers," J. Imaging Sci. Technol., 55(1), 10505-1-10505-16 (2011).

[17] Qu, Y. and Gooran, S., "Simple color prediction model based on CIEXYZ using an effective coverage map," J. Imaging Sci. Technol., 56(1), 0105061-105069 (2012).

[18] Qu, Y. and Gooran, S., "Investigating the possibility of using fewer training samples -- in the color prediction model based on CIEXYZ using an effective coverage map," Proc. CGIV 2012 (6th European Conference on Color in Graphics, Imaging, and Vision), 163-168 (2012).

[19] Qu, Y. and Gooran, S., "Simple spectral color prediction model using multiple characterization curves," Proc. TAGA (Technical Association of the Graphic Arts), Portland, Oregon, USA. (2013).

[20] Qu, Y., [Color prediction and separation models in printing-Minimizing the colorimetric and spectral differences employing multiple characterization curves] PhD Dissertation No.1540, Linköping Studies in Science and Technology (2013). 